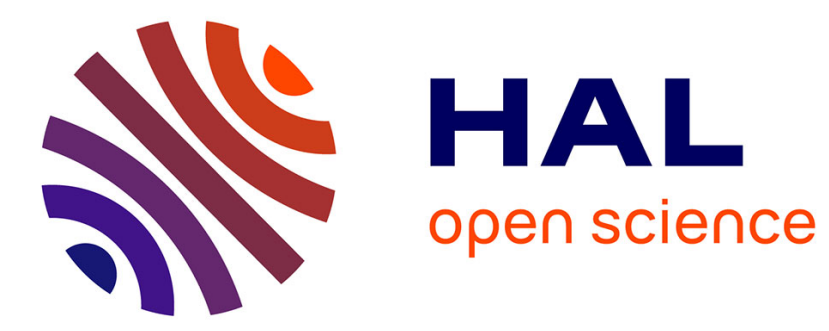

\title{
Les cours de préparation à la naissance comme espace de ségrégation sexuelle
}

\author{
Béatrice Jacques
}

\section{To cite this version:}

Béatrice Jacques. Les cours de préparation à la naissance comme espace de ségrégation sexuelle. L'Information géographique, 2012, 76, pp.108-121. 10.3917/lig.762.0108 . halshs-00720955

\section{HAL Id: halshs-00720955 \\ https://shs.hal.science/halshs-00720955}

Submitted on 20 Jan 2022

HAL is a multi-disciplinary open access archive for the deposit and dissemination of scientific research documents, whether they are published or not. The documents may come from teaching and research institutions in France or abroad, or from public or private research centers.
L'archive ouverte pluridisciplinaire HAL, est destinée au dépôt et à la diffusion de documents scientifiques de niveau recherche, publiés ou non, émanant des établissements d'enseignement et de recherche français ou étrangers, des laboratoires publics ou privés. 


\title{
Les cours de préparation à la naissance comme espace de ségrégation sexuelle ${ }^{1}$
}

\author{
Par Béatrice Jacques
}

Béatrice Jacques, MCF sociologie, Centre Émile-Durkheim UMR 5116, Université Bordeaux Segalen

beatrice_jacques@yahoo.fr

Les cours de préparation à la naissance constituent un espace de rencontres, d'échanges entre patientes, unique à l'hôpital ${ }^{2}$. Aucune autre spécialité médicale, offre dans sa prise en charge un lieu spécifique, institutionnel, organisé, où on parle de la "pathologie », où différents experts viennent donner des conseils, des explications sur la «maladie », où on se retrouve entre patients. En effet, si les relations médecins-patients fonctionnent selon un cadre normatif, les rapports entre malades ne font l'objet, à l'hôpital, d'aucune règle, d'aucun code préexistant à leur rencontre. L'institution hospitalière, de façon générale, n'a pas pensé le lien entre malades.

Depuis 1959, huit séances de préparation à la naissance ${ }^{3}$ sont remboursées par la sécurité sociale. L'institutionnalisation de la préparation est directement liée au travail du Docteur F. Lamaze, à la clinique des Bluets à Paris. En 1951, ce médecin accoucheur, au cours d'un voyage en URSS, découvre l'accouchement sans douleur (ASD) mis en place par le professeur Nicolaïev. Cette méthode,

\footnotetext{
1. Cette communication présente les résultats d'un travail de doctorat de sociologie publié en 2007 (Jacques) qui portait plus largement sur l'expérience de la grossesse et de l'accouchement en France.

2. La dernière enquête de périnatalité réalisée en 2003, montre que 66,60\% (contre 69,70\% en 98) des femmes enceintes primigestes (femmes enceintes pour la première fois) ont suivi une préparation à la naissance et seulement 24,90 \% des multipares (femmes qui ont déjà accouché d'un ou plusieurs enfants), soit un taux de participation de $45,75 \%$. Pour les $\frac{3}{4}$ des parturientes, les préparations ont été réalisées dans la maternité où elles ont accouché. Plusieurs enquêtes ont déjà montré, que plus les femmes enceintes ont un faible niveau social (le plus souvent sans emploi) et plus elles ont une origine étrangère, moins elles assistent aux cours de préparation. Enquête nationale périnatale 2003. Compléments de cadrage : les disparités sociales en matière de santé périnatale et apports des autres sources, Ministère des Solidarités, de la Santé et de la Famille, février 2005.

Les femmes que nous avons pu observer pendant les cours de préparation à la naissance renvoient à ces différentes caractéristiques. Dans le service du CHU, à partir d'un relevé systématique des femmes inscrites pendant un an, nous avons pu établir que presque la moitié des femmes issues de la classe moyenne, assiste à la préparation, alors que c'est seulement le cas, pour un peu plus d'un tiers des parturientes d'origine populaire. Ainsi, les termes de femmes, futures mères et pères employés dans le texte, feront principalement référence à ce groupe de femmes (et leurs conjoints) appartenant en majorité à la classe moyenne.
}

3. On peut s'y inscrire à partir du $6^{\mathrm{e}}$ mois de grossesse. 
fondée sur la théorie de Pavlov, repose sur l'idée qu'il faut, par une action pédagogique, enlever la peur de l'accouchement. Dès son retour en France, Lamaze réalise son premier accouchement sans douleur et généralise la préparation à l'ensemble des femmes de la clinique des Bluets. «L'idée qui sous-tend cette préparation, c'est de donner une éducation rationnelle » (Leulliez, 2002:7) aux parturientes. Beaucoup de sages-femmes sont venues se former auprès de l'équipe des Bluets, Lamaze a lui-même parcouru la France pour diffuser sa méthode. En 1959, la reconnaissance par la sécurité sociale, permet l'ouverture de cours de PPO (psycho-prophylaxie-obstétricale) dans de nombreux établissements hospitaliers.

Mais très vite, dans le milieu des années soixante, l'ASD reçoit de nombreuses critiques, plus précisément de la part de la psychanalyse, qui reproche aux continuateurs de Lamaze d'utiliser la suggestion dans la relation médecin-patient et de ne pas assez tenir compte de la psyché, de la complexité des situations individuelles. Le courant de l'humanisation, dans les années soixante-dix, met en cause de nouveau les cours de PPO. Le Dr M. Odent, qui prône une naissance écologique, conteste l'idée du pouvoir de la science sur laquelle repose toute la théorie lamazienne. En 1982, on dénombre encore $58 \%$ de femmes qui accouchent avec une préparation à l'ASD. Mais déjà, l'analgésie péridurale introduite dans les années soixante-dix en France, fait son effet auprès des femmes. Progressivement, les termes d'ASD et de psychoprophylaxie disparaissent au profit de préparation à la naissance. Désormais, les cours ne se concentrent plus sur la maitrise de la douleur, celle-ci ayant « techniquement » disparue avec la péridurale, mais sur un accompagnement physiologique et psychique de la grossesse. Il s'agit moins de préparer à l'accouchement, mais de suivre les femmes dans leur transformation, de leur permettre de pouvoir poser plus facilement des questions, de leur offrir un soutien psycho-affectif.

Le travail d'observation dans plusieurs services de maternités ${ }^{4}$, a rapidement montré que cet espace de la préparation ne pouvait être considéré par les professionnels de la naissance et par les parturientes que comme un lieu féminin, comme la reconstruction du gynécée. Ce terme nous renvoie à la société grecque antique où l'espace était fortement ségrégué sexuellement. Le foyer, l'intérieur étaient des zones exclusivement féminines et tout ce qui relevait de taches intellectuelles, culturelles, était de l'ordre du masculin. L'espace du gynécée est intéressant pour notre travail car il permet de comprendre pourquoi ces séances de préparation se présentent et s'organisent comme un lieu fortement

\footnotetext{
4. Pour être la plus exhaustive sur l'ensemble des expériences possibles, sur l'ensemble des contextes, 3 niveaux de maternité ont été étudiés (définis selon le plateau technique et les professionnels disponibles) dans des structures de type différent, un service d'un CHU (niveau 3), un service d'une clinique privée (niveau 2) et un service d'une petite maternité de proximité (niveau 1), présentant chacun des particularités sociologiques dans leur population. Des femmes qui ont fait le choix d'accoucher chez elles, ont aussi été rencontrées. Deux méthodes d'enquêtes ont été principalement utilisées : l'observation et l'entretien.
} 
genré. Nous nous demanderons dans un premier temps, comment les patientes se représentent et utilisent cet espace féminin. Si nous montrerons que la modernité a déplacé le rôle originel du gynécée, comme temps collectif de partage d'expériences et de transmission de savoirs, en temps narcissique, nous constaterons par ailleurs que la question de l'entrée et de la place des pères dans la maternité n'est pas réglée. Alors même qu'ils sont désormais exhortés à participer à tous les événements de la grossesse et de l'accouchement et que toute absence fait l'objet d'une réprobation collective (de la part des parturientes et des professionnels de soins hommes et femmes), nous verrons dans un deuxième temps, que l'institution hospitalière mais plus largement notre société, participent à la production d'un impensé paternel, qui empêche les pères de pouvoir jouer totalement le rôle que l'on attend d'eux.

\section{- La préparation comme gynécée : un espace au service de la femme, du groupe de femmes}

L'observation des rencontres prénatales dans les structures étudiées, met en évidence le postulat de base sur lequel elles reposent. L'approche descriptive et l'apprentissage «scientifique » développés, sont fondés sur la croyance que l'information permet la connaissance. Plus la femme peut appréhender rationnellement l'événement biologique moins elle a peur, moins elle est angoissée. Plus la femme connaît le fonctionnement de son corps, mieux elle va accoucher.

Concrètement, les séances de préparation remplissent plusieurs fonctions. Tout d'abord, elles doivent permettre de répondre aux questions des femmes. La préparation remplit un rôle purement organisationnel, inscrit dans la division du travail médical qui prend ici un aspect sexué. Le médecin ${ }^{5}$, axé sur la dimension physique, pathologique de la grossesse, délègue à la sage-femme ${ }^{6}$ tout ce qui concerne les interrogations « anodines », « normales », « existentielles » des parturientes. « La préparation est importante pour leur expliquer comment ça va se passer, pour leur expliquer les petits trucs normaux de la grossesse, pour qu'elles parlent d'elles. Nous, on n'a pas le temps de le faire en consultation » confie le Docteur M. (55 ans, maternité de niveau 2). On retrouve de façon assez classique la division : homme-rationalité/femme-subjectivité. Le

\footnotetext{
5. Pendant l'enquête, seule une femme gynécologue-obstétricien a été rencontrée parmi les 11 médecins interviewés. Contrairement aux gynécologues médicaux (90\% de femmes), la spécialité de l'obstétrique est à dominante masculine (60,3\% en 2012). Sa composante chirurgicale semble en être une des explications. INSEE, « Médecins suivant le statut et la spécialité en 2012 », Tableaux de l'économie française, 2012 ; Bessière Sabine, «La féminisation des professions de santé en France : données de cadrage », RFAS, 2005-1, p. 19-33.

6. 98,1\% des sages-femmes sont des femmes en 2011. DRESS, « Les professions de santé au $1^{\text {er }}$ janvier $2011 »$, Séries Statistiques, n 158, juillet 2011.
} 
rôle des sages-femmes, c'est ainsi qu'elles le définissent, est d'offrir un soutien affectif, un espace de parole. Les sages-femmes de la préparation se présentent d'ailleurs comme des spécialistes de la relation d'écoute. Dans ce cas précis, cette séparation des rôles, n'est pas vécue sur le versant négatif de la domination masculine. Deux des trois sages-femmes responsables de la préparation (des trois maternités enquêtées) se sont formées à la sophrologie et à la relaxation pour développer « une approche du corps à travers les émotions et les sensations » (K., 55 ans, maternité de niveau 2). Cependant, nombreuses sont les femmes qui font part de leur difficulté à prendre la parole devant un groupe de 20 personnes ou face à un expert. Les parturientes expliquent leur impossibilité, non seulement de confier leurs problèmes personnels devant le groupe, mais aussi à la sage-femme, qu'elles n'ont finalement pas l'occasion de rencontrer de façon individuelle. Enfin, la préparation sert à acquérir des connaissances sur soi et l'enfant. Cette fonction intervient seulement en deuxième position dans les discours des femmes, alors qu'elle est présentée comme la fonction première des cours par les professionnelles. Lors des séances, la sage-femme, à l'aide d'un mannequin ou de planches anatomiques, explique aux femmes la croissance du fœetus, son positionnement dans l'utérus et le moment de l'expulsion. Elle montre également des postures pour soulager les maux de la grossesse, explique comment respirer entre deux contractions. Alors que c'est sur la diffusion de ce savoir «théorique » que l'existence des séances de préparation repose (et sont remboursées), alors que c'est ce côté qui est le plus valorisant pour les sages-femmes, nous allons voir, que les femmes parce qu'elles sont déjà sensibilisées, attendent autre chose de la préparation. Elles espèrent pouvoir y trouver tout ce qu'elles n'ont pas pu obtenir dans la relation au médecin, une attention particularisée.

\section{La préparation comme temps pour soi (pour la femme enceinte et non le couple) ${ }^{7}$ et entre soi}

La préparation est avant tout présentée par les professionnels et par les femmes comme un temps privilégié qui leur est proposé pour prendre soin d'elles, de leur corps, pour mieux se connaître et s'appréhender pendant leur grossesse. Pour Lucie (maternité de niveau 2) qui suit la préparation classique de la maternité, «c'est une manière de profiter de soi et de sa grossesse ». Les cours de relaxation souvent cités par les femmes, sont vus comme un temps de repli sur soi, d'introspection. Les exercices de gymnastique douce ou de yoga ont comme objectif pour les sages-femmes, «que la femme se retrouve et se pose le temps de sa grossesse pour travailler le lien avec l'enfant » (C.,

7. Nous parlerons ici exclusivement des préparations classiques proposées encore majoritairement dans les maternités. D'autres types de préparation comme l'haptonomie, demandent une participation du père plus importante et proposent une réflexion sur la place de l'homme pendant la grossesse et l'accouchement. 
45 ans, maternité de niveau 1). Ce doit être un temps pour la femme, pour se préparer tant physiquement que psychiquement. D'une certaine manière, pour les professionnels, c'est une façon pour la parturiente de commencer à s'occuper de son enfant, à se préparer à entrer dans son rôle de mère. On ne rencontre pas d'espace équivalent pour les hommes.

Les séances ne sont jamais vécues comme une contrainte, « on aime parler de soi » dit Bénédicte (maternité de niveau 2). Ce temps de préparation est vécu comme une parenthèse dans la vie quotidienne, mais aussi dans le suivi médical. L'ambiance détendue du cours de préparation, le temps laissé pour répondre aux questions de chacune, contrastent avec le temps de la consultation. Pour certaines, c'est l'occasion de découvrir des techniques de rapport à l'autre et à soi. La sophrologie permet par exemple, une forme de déconnexion d'avec le corps. Pour la sage-femme qui pratique cette méthode, elle peut dans le cadre de l'accouchement atténuer la douleur et permet un travail plus large sur la psyché. Chacune des patientes qui a choisi la technique de la sophrologie, revient sur l'importance du travail sur elle-même qu'elle a pu effectuer, avant même d'aborder la préparation à l'accouchement. Par exemple, Dominique raconte qu'elle a enfin pu parler de la relation avec sa mère. « J'avais jamais fait ce travail qui est pourtant essentiel au moment de la grossesse. Ca m'a permis de me libérer, d'être mieux » (maternité de niveau 2).

Psychologues et psychanalystes ont, depuis longtemps, montré que la période de la grossesse est un temps où l'on souhaite parler de soi, régler les difficultés personnelles et familiales. Beaucoup de parturientes ont regretté de n'avoir pas trouvé le lieu, le temps ou la personne dans la structure d'accueil pour se raconter. Certaines se questionnent sur l'absence d'une prise en charge psychologique pendant la grossesse. Souvent, nous avons dû nous-mêmes, jouer ce rôle d'écoute et transformer l'entretien sociologique pour permettre l'expression des femmes sur des conflits familiaux, des problèmes conjugaux, des avortements cachés, des relations extraconjugales, des problèmes de sexualité dans le couple... non confiés au médecin ou à la sage-femme. L'envie de dire, de se raconter semble exacerbée pendant la grossesse, par ce passage de statut de fille à celui de mère. C'est un travail psychique qui doit se faire en situation de face à face, ce que ne permet pas l'organisation actuelle des cours de préparation.

\section{Le difficile partage de l'expérience}

Pour les parturientes interrogées, un autre des principaux intérêts de la préparation est de pouvoir rencontrer d'autres femmes enceintes, des pairs et de partager les expériences. «Les autres mamans nous apprennent des choses » (France, maternité de niveau 2), «C'est vraiment important car on n'a pas toutes le même âge, certaines ont déjà des enfants, elles nous racontent, c'est 
rassurant » (Céline, maternité de niveau 1), «C'est bien de partager entre nous, parce qu'on est un petit peu isolé finalement » (Irène, maternité de niveau 1). La nécessité de la reconstitution originelle du groupe de femmes est présente dans tous les discours. Les médecins, eux-mêmes, insistent sur la particularité de la préparation comme lieu exclusivement féminin. « Ca ne peut être fait que par une sage-femme, femme. Il faut qu'elles se retrouvent entre elles, qu'elles se racontent leurs trucs de femmes » (Dr V., 33 ans, maternité de niveau 3).

Pour certaines d'ailleurs, la féminisation du groupe est ce qui facilite la prise de parole, («c'est plus facile de poser des questions car on est entre femmes, on se sent plus à l'aise » (Sophie, maternité de niveau 3)) et joue un rôle de réassurance mutuelle ( $«$ on voit que tout le monde se pose les mêmes questions, qu'on est dans la norme, c'est rassurant » (Patricia, maternité de niveau 2)). Pour d'autres, la préparation permet de rencontrer d'autres parturientes dont le terme est proche et qu'elles vont retrouver en salle d'accouchement et en suites de couches. Des amitiés peuvent se créer, même si le plus souvent, elles ne durent que le temps de l'hospitalisation. La préparation se présente comme un lieu de socialisation. C'est bien là, une particularité de l'obstétrique à l'hôpital. Alors que plusieurs auteurs montrent la difficulté dont témoignent généralement les patients, à rencontrer, à séjourner dans la même chambre qu'un autre malade, qui leur renvoie leur propre image de la souffrance, de la déchéance ou de la mort, la naissance, moment heureux par excellence, donne envie aux femmes de se rencontrer, d'échanger. Alors qu'ailleurs, on fuit les autres patients, ici, l'autre devient une ressource. Il renvoie l'image positive du ventre rond, à laquelle on veut s'identifier.

Cependant, si toutes les femmes qui assistent à la préparation sont unanimes sur le désir de partager, de discuter entre elles, plusieurs évoquent le peu de place laissé par la sage-femme animatrice, à l'échange d'expériences dans les séances. «Les échanges sont inexistants entre mamans. On est toutes assises là, personne ne parle, sauf à la première séance où on devait se présenter. Il faudrait que ça dure plus longtemps et qu'on se connaisse mieux. La sage-femme fait pas trop ce boulot-là. Il n'y a pas de dynamique de groupe » (Bénédicte, maternité de niveau 2). «C'est dommage que les échanges entre femmes soient stoppés pour faire les exercices. Ce serait bien qu'il y ait une séance pour ça. Ca fait du bien d'entendre ce que les autres disent, ressentent » (Katia, maternité de niveau 3).

Pour les professionnelles, le contenu théorique du cours est plus pertinent que l'échange d'expériences. Quand il est possible, il est lui-même encadré, « sous surveillance ». Il se fait devant un professionnel qui peut intervenir pour corriger tous propos scientifiquement erronés. La place pour l'échange de savoir profane n'est en tout cas pas celle des cours de préparation. En fait, les femmes qui ont suivi la préparation classique proposée par les structures, sont moins déçues par le contenu des cours ( « de toute façon, explique Rabia, on oublie tout, le jour 
même » (maternité de niveau 3)), que par l'absence de diffusion d'un savoir féminin qui fait l'intérêt de la reconstruction du gynécée.

\section{- Quelle place pour le père ?}

Dès les années soixante-dix, sous l'impulsion du courant de l'humanisation, le père est, non seulement invité à assister à l'accouchement, mais aussi aux consultations et à la préparation à la naissance. Déjà, dans les années cinquante, F. Lamaze demandait aux futurs papas d'être présents pendant la grossesse et à l'accouchement. Nos observations et le témoignage des femmes montrent une faible participation des hommes aux séances de préparation (cinq conjoints (1 au CHU, 3 à la maternité de niveau 2, 1 à la maternité de niveau 1) ont accompagné leurs femmes parmi les 60 parturientes interrogées). Eux-mêmes témoignent de leur difficulté à s'intégrer dans ce groupe de femmes. «Je suis venu à la première séance et quand j'ai vu qu'il y avait que des nanas, je suis reparti » explique Christophe, conjoint de Guenaëlle, (maternité de niveau 2). Jean, (maternité de niveau 3) raconte, lui, ses réticences « à poser des questions devant toutes ces futures mamans ». Le monde de la naissance leur semble exclusivement féminin, même si le comportement que l'on attend de l'homme est largement évoqué dans les séances. Il doit être présent pendant la grossesse, soutenir sa femme, assister à toutes les échographies, l'aider à pousser lors de l'accouchement, couper le cordon, s'occuper des autres enfants pendant le séjour de la femme à l'hôpital et préparer son retour. Toutes les femmes interviewées souhaitent la présence de leur mari lors de l'accouchement. L'homme aurait en fait peu le choix aujourd'hui : sa participation est devenue un fait culturel. D'ailleurs depuis quelques années, les recherches et publications scientifiques et de vulgarisation sur la « grossesse et l'accouchement » du père et plus généralement sur la paternité, se sont multipliés. Comme le signale P. Ben Soussan (2003), il n'est désormais plus possible de lire un magazine spécialisé, qui s'adresse principalement à un public féminin, sans y trouver un article, un témoignage sur le travail de paternité. Une analyse rapide des articles de journaux spécialisés montre que les auteurs choisissent de traiter le sujet essentiellement selon deux modes d'écriture : la critique du paterfamilias et la vulnérabilité du père moderne. Jusqu'à la fin des années soixante-dix, les futurs parents sont présentés selon le modèle classique de la division des rôles sexuels. Au travers de la dénonciation de ce schéma familial par les féministes, la psychologie s'empare de la figure du père et multiplie les travaux pour insister sur la pauvreté d'une relation basée exclusivement sur l'autorité. Jusque-là, le concept lacanien de Nom-du-Père, domine la pensée et empêche de reconnaître au père une place dans la dyade mère-enfant. Pour Lacan, de par son origine physiologique, la relation fusionnelle mère-enfant n'a aucun équivalent chez le père. C'est la mère qui dirige le regard de l'enfant, c'est donc elle qui décide 
si elle veut introduire un tiers dans leur relation. Selon la théorie lacanienne, il n'y a de place pour l'homme que celle reçue de la femme. C'est elle seule, qui distribue les rôles respectifs et bien distincts du père et de la mère. Ainsi, jusqu'à une période récente, la littérature de vulgarisation s'est caractérisée par un discours où le père prenait une place tardive (à partir de 3 ans) dans la socialisation de l'enfant. Il est de plus décrit, comme peu présent dans le foyer, plus préoccupé par son activité professionnelle que par son rôle éducatif.

Puis, progressivement l'image paternelle change. Les magazines montrent de plus en plus, prenant un congé pour garder l'enfant malade, baignant le bébé, le câlinant ${ }^{8}$. Chaque revue annonce l'arrivée du «nouveau père », même si chaque époque a eu ses «nouveaux pères ». Pour F. Hurstel, il y a toujours eu un avant et un après dans l'imaginaire collectif autour de la question du père. «La mutation paternelle ne date pas d'hier, depuis la révolution française elle s'inscrit dans les lois (doublée d'une approche psychanalytique) modifiant graduellement et de façon irréversible, à la fois la place sociale du père et l'image que tout un chacun s'en fait ${ }^{9}$. Fait nouveau cette fois-ci, l'homme ne s'intéresse plus à l'enfant seulement une fois qu'il est né, on sollicite sa présence et son attention dès l'annonce par la femme de sa grossesse. Désormais, comme pour la mère, l'homme a neuf mois pour être père.

Des cours de préparation exclusivement pour les pères sont proposés à la maternité de niveau 3 dans laquelle nous avons enquêté. Un obstétricien et une sage-femme sont présents pour répondre aux questions des futurs papas qui participent aux séances le plus souvent sur l'injonction de leur compagne. On peut remarquer que ces derniers, axent essentiellement leurs questionnements sur l'accouchement et l'après-accouchement, comme si la période de la gestation, qu'ils ne peuvent vivre que par procuration, les concernait moins. On peut classer leurs interrogations selon trois grandes catégories : les questions sur la maîtrise technique de l'événement : « Comment reconnait-on le moment ?, Le déclenchement, comment ça se passe ?, Combien de temps dure l'accouchement ?...»; les questions sur le rôle : « Expliquez-nous les deux, trois choses à faire et à ne pas faire pendant l'accouchement ?, Faut-il absolument couper le cordon ?, Apprend-on au père à faire la toilette ? Le père doit-il toujours avoir ce côté sanction dans l'éducation ?...»; les questions sur le désir et la sexualité : «Quand peut-on reprendre les relations sexuelles ?, Où va le sperme pendant la grossesse ?, Les rapports sexuels peuvent-ils causer des contractions ?, Quand le ventre de la mère redevient-il normal ?... ». Les interrogations des pères sont de fait, assez proches de celles des mères. Mais comme le note $\mathrm{A}$. Benoît, ce

8. Même si l'on peut penser comme le disent G. Delaisi de Parceval et S. Lallemand que « les nouveaux pères en question n'ont pas attendu le feu vert des manuels pour « materner » leurs bébés », Delaisi De Parceval G., Lallemand S., L'art d'accommoder les bébés, Paris, Odile Jacob, 1998 (1980), p. 71.

9. Hurstel F., La déchirure paternelle, Paris, PUF, 1996, p. 120. 
qui est le plus significatif dans ses séances, c'est que « les hommes viennent dire avant toutes choses qu'ils ne se sentent pas pères du tout » (2003: 59). Comment ressentir psychiquement et corporellement ce fotus, ce futur enfant qu'on ne porte pas, comment devenir père « du ventre de sa compagne »? L'homme ne peut vivre la grossesse que par procuration, que par ce que la femme veut bien partager ou l'oblige à incarner.

\section{L'espace de la maternité comme " aire de ségrégation » sexuelle}

Dans une étude réalisée sur « La paternité en maternité » (2006), Gérôme Truc parle d'institution matrifocalisée pour décrire la place des hommes dans la structure de soins. Il travaille plus précisément sur le service d'accueil des accouchées et montre que les pères ne peuvent incarner dans cet espace exclusivement féminin, qu'un répertoire de rôles restreint et très codifié. La posture dominante est celle de la discrétion, voire de l'invisibilité (« se faire oublier »). Ici le père a parfaitement bien compris que ce qu'on attend de lui, est qu'il limite ses déplacements à l'espace circonscrit de la chambre mais aussi qu'il sache en sortir quand sa présence est jugée inopportune, gênante pour le bon fonctionnement des soins. Le couloir, qui pourrait devenir un lieu d'échange entre «pairs », ne peut lui non plus être investi trop longuement. Le père n'a non seulement pas d'espace propre et lorsqu'il intègre les zones féminines et soignantes, il est préférable qu'il passe inaperçu. Sa seule possibilité d'expression du rôle passe par le mandat de la femme. Ses déplacements deviennent alors légitimes dès lors qu'il est accompagné par la mère. Quand il est circonscrit à la chambre, tous les actes qui lui sont confiés, sont parfaitement codifiés et « sous surveillance ». Mais ce qu'on attend avant tout de l'homme, c'est qu'il prenne soin de sa conjointe «c'est à ce niveau qu'il est le plus spécifiquement "utile" dans et à la maternité » (Truc : 2006 : 344). Cela montre à la fois la pauvreté de la réflexion de l'hôpital et des professionnels de santé sur la place à lui accorder et plus largement l'incapacité de notre société à repenser son rôle. Une autre enquête ${ }^{10}$ citée dans un article de Danielle Boyer et Benoît Céroux montre bien que « la localisation des espaces réservés aux enfants dans des lieux féminins (aires de jeux, entrées et sorties des écoles, rayons de puériculture des magasins, etc.) [...] (peut rendre) la présence de l'homme singulière » (2010: 55), voire incongrue. Les femmes elles mêmes, sous l'effet d'une socialisation genrée, rencontrent des difficultés, voir refusent d'intégrer toute présence masculine dans des territoires qui se présentent encore comme des lieux à préserver ${ }^{11}$.

10. Merla L., « Pères au foyer : une expérience « hors normes » », Recherches et prévisions, n 90, 2007, p. 17-27.

11. Tel ce père interviewé par D. Boyer et B. Céroux qui explique que si les autres mères et l'institutrice apprécient qu'il vienne chercher ses enfants à la sortie de l'école, on lui signifie par ailleurs que sa présence au voyage scolaire serait malvenue. Boyer D., Céroux B., « Les limites des politiques publiques de soutien à la paternité », Travail, genre et sociétés, novembre 2010 ( $\left.\mathrm{n}^{\circ} 24\right)$, p. 47-62. 


\section{Pour une lecture anthropologique de l'impensé paternel}

L'anthropologie a bien montré que selon les sociétés, la gestation et l'accouchement peuvent être un moment exclusivement maternel, exclusivement paternel (rôle symbolique du père) ou exclusivement social (l'enfant est avant tout accueilli par un groupe). Les mythes fondateurs ont été les premiers à exclure la femme de l'enfantement tel Athéna sortant du front de Zeus. Pour la culture judéo-chrétienne, la création de la femme est bien une véritable naissance par accouchement costal masculin. Ève naît de la côte d'Adam. Pourtant, les représentations occidentales actuelles de la grossesse et de l'accouchement sont totalement féminines.

\section{«La relation se fait d'abord avec la mère »}

C'est sous l'influence de travaux de la psychologie, qui insistent sur l'importance du père pendant la grossesse et dans les soins au bébé, que les professionnels de santé vont ouvrir progressivement les portes de la maternité aux hommes. Ce sont les sages-femmes, proches des mouvements féministes et imprégnées d'un «discours psychologique » qui associent, les premières, les pères à l'événement de la naissance. Puis très vite, les hommes sont vivement incités à participer, non seulement à l'accouchement, mais à toute la grossesse. Les conseils se multiplient. Les psychanalystes se divisent, entre ceux pour qui le père doit autant materner, participer aux soins que la mère ${ }^{12}$ et ceux comme F. Dolto, qui pensent que « ce n'est pas par le contact physique, mais par la parole que les hommes peuvent se faire aimer d'affection et respecter de leurs enfants » (1977: 171). Si le débat passionne les intellectuels, sur le terrain, les professionnels de santé et les futurs parents ont eux parfaitement intégré l'idée de la présence du père pendant la grossesse et à l'accouchement, jusqu'à en faire une norme culturelle. À partir de la fin des années quatre-vingt, chaque homme vit sa présence comme une obligation, le personnel soignant allant jusqu'à porter l'opprobre sur tout conjoint qui aurait fui une des étapes essentielles d'entrée dans son rôle de père.

Si le père trouve aussi peu sa place à l'hôpital et/ou se reconnaît peu dans les rôles qui lui sont attribués, c'est que nous assistons dans la culture occidentale à « un déni de la paternité » (Delaisi de Parceval, 1981). Pour G. Delaisi de Parseval, notre société, totalement tournée vers la relation maternelle, a amputé l'homme d'une part de sa paternité. Toutes les théories psychanalytiques occidentales, qui font de la grossesse et de l'accouchement « des moments essentiels et « indélébiles » dans la relation mère-enfant» (1981:296), constituent un obstacle au sentiment paternel. Même si une place de plus en plus importante tend à être accordée au père, si on l'incite à être acteur dans la grossesse et 
l'accouchement, la figure centrale de l'attachement reste celle de la mère. On retrouve cette interprétation dans les discours des parturientes et des professionnels. «La relation se fait d'abord avec la mère. Le père, il est là, il fait ce qu'il peut. Il doit attendre » (Dr V., 33 ans, maternité de niveau 3). Or « il n'y a en réalité nulle inégalité entre homme et femme devant la procréation ; la différence et les avantages supposés d'un sexe par rapport à l'autre, dans quelques sens qu'ils se manifestent, sont construits, fruits de facteurs idéologiques et culturels » (Delaisi de Parceval, 1981: 288). La femme et l'homme, vivent tous deux « psychiquement » la grossesse et l'accouchement. Ils produisent des angoisses, investissent leur rôle mais sur un mode différent. Pour preuve, les manifestations psychosomatiques que produisent les pères pendant la grossesse et au moment de l'accouchement que l'anthropologie nomme la couvade. La couvade, dans les sociétés dites exotiques, consiste à faire respecter au père des rituels de protection pendant la grossesse et l'accouchement.

\section{Une couvade non avouable}

G. Delaisi De Parceval qui a étudié la « couvade européenne », note qu'elle est depuis longtemps pratiquée. Les premières descriptions datent du Moyen-Âge. Elle définit ce rite comme « des manifestations psychosomatiques individualisées, une volonté inconsciente de participation du père au rôle de la mère » (1981 : 69). Selon l'auteur, le « tabou de la paternité », empêche l'homme (le primo-père particulièrement) de se confier sur les troubles psychiques que produit sur lui l'annonce de l'arrivée d'un enfant, de pouvoir avouer « la jalousie » qu'il ressent à l'égard de toutes les attentions concentrées sur sa femme et l'enfant à venir. Ces sentiments non exprimés, vont alors se manifester, le plus couramment de façon corporelle ou sociale. On a ici affaire essentiellement à des « couvades à bas bruits, non perçues comme telles par les intéressés et leur entourage, tant il est vrai que ces manifestations, n'étant pas culturellement admises, ne sont pas recevables au niveau conscient » (Delaisi de Parceval, 1981 : 79).

Les manifestations corporelles renvoient le plus souvent au modèle médical soustractif décrits par Laplantine (1992). En effet, certains pères développent des pathologies bénignes qui se manifestent par un gonflement, une excroissance (qui rappelle l'évolution du corps de la femme enceinte), qu'il va falloir extraire ou soigner. On trouve ainsi chez les futurs pères, des troubles ORL liés au gonflement des amygdales, des troubles oculaires (orgelet), des problèmes dentaires (extraction). Une deuxième catégorie de symptômes est également courante chez les primo-pères. Certains hommes présentent tous les maux, généralement associés à l'état de grossesse : prise de poids, insomnies, troubles digestifs, nausées, maux de dos. Le deuxième type de comportements liés à la couvade, renvoie à ce qu'on nomme les « acting de la paternité ». Les futurs pères commettraient particulièrement dans cette période de la maternité et de 
l'accouchement, des actes considérés comme déviants. Trois types de passage à l'acte sont les plus fréquents : les accidents (circulation, sport...), les fugues (les hommes se réfugient dans le travail, voyages d'affaire...), un changement de sexualité (homosexualité, adultère...). Pour la psychanalyse, ces passages à l'acte permettent de détourner l'attention du groupe familial, focalisée sur la femme enceinte, vers le futur papa. Il n'est donc pas « absurde de supposer que si le père ne porte pas l'enfant dans son ventre, il peut, tel Zeus pour sa fille Athéna, le porter dans sa tête » (Delaisi de Parceval, 1981 : 19). Ainsi les hommes, tout comme les femmes, effectuent un travail psychologique pendant la grossesse, qui leur permet d'entrer déjà dans la paternité. Ces phénomènes de couvade, même s'ils sont connus par les soignants, ne sont pas considérés comme tels et se racontent en salle de garde sur le mode de « la bonne blague ». Les cours de préparation pour les pères ne les évoquent pas non plus. Alors que la légitimité de la place du père, au même titre que la mère, est de plus en plus affirmée par de nombreux chercheurs de différentes disciplines, on assiste à une certaine inertie du modèle culturel. La manifestation « symbolique » d'une paternité en devenir est encore socialement non reconnue.

\section{Le père idéal ?}

L’Europe, a permis ces dernières années d'impulser, chez les États membres et plus particulièrement en France, une réflexion sur l'implication des pères dans la vie familiale. Très concrètement en janvier 2002, l'État français met en place un congé paternité d'une durée de 11 jours, sous-tendu à la fois par un impératif d'égalité homme/femme ${ }^{13}$ et par des nouveaux questionnements sur la place et le rôle des pères.

Il semble qu'aujourd'hui les règles qui encadrent le rôle du père soient multiples et parfois contradictoires. «Les pères n'occupent pas à l'égard des touts petits une position facile à tenir du simple fait que celle-ci n'est (pas) véritablement définie par un discours social qui ferait consensus » précise G. Neyrand (2000 : 250). C'est un peu comme si chacun, en était réduit à l'inventer dans le cadre de sa vie personnelle, de son histoire avec son propre père et avec ses propres ressources. Il existe deux modèles dont il faut cependant, à tout prix se démarquer : le père « sévère » (autorité du paterfamilias, dont G. Neyrand nous rappelle la progressive disparition au profit de l'État et de la mère) et le père « maternisé » (l'homme qui materne est toujours suspecté de dévirilisation même si l'on sait aujourd'hui que les pères impliqués dans les soins de l'enfant

13. « La participation équilibrée des femmes et des hommes au marché du travail et à la vie familiale, qui est un avantage tant pour les hommes que pour les femmes, constitue un élément indispensable au bon développement de la société et la maternité, la paternité, ainsi que les droits des enfants, sont des valeurs sociales éminentes qui doivent être sauvegardées par la société, par les États membres et par la Communauté européenne » : extrait du texte de résolution du Conseil européen des Affaires sociales du 29 juin 2000 in Boyer D., Céroux B., «Les limites des politiques publiques de soutien à la paternité », Travail, genre et sociétés, novembre 2010 ( $\left.\mathrm{n}^{\circ} 24\right)$, p. 57-58. 
développent des attitudes propres qui les distinguent bien de la mère). Par ailleurs, les enquêtes de l'Insee ou de l'Ined $^{14}$ le montrent régulièrement, le temps de participation des hommes aux taches domestiques et de soins aux enfants est encore très limité. Il y aurait donc aujourd'hui plusieurs expressions de la paternité, plusieurs façons de l'incarner. On comprend ici que l'homme qui veut pleinement jouer son rôle parental, doit faire à la fois avec un discours sur le caractère incontournable de la légitimité paternelle dans le travail parental, et des pratiques sociales qui restent éloignées de ce modèle.

\section{- Conclusion}

Si le père est aujourd'hui sollicité à la maternité, l'institution n'a pas encore défini le rôle qu'il devait y jouer. Sa position est très paradoxale, il doit être là mais il est en même temps déresponsabilisé car tout ce qui relève de la grossesse ou des soins à l'enfant est renvoyé à la mère. On lui demande de prendre une place alors même qu'aucune des composantes du rôle de père ne peut s'exprimer. Tout se passe aujourd'hui, comme s'il ne pouvait incarner sa fonction paternelle que contre l'institution.

Si de récentes enquêtes montrent à la fois que les figures de la paternité semblent désormais moins prescrites, que les hommes ont une marge de négociation dans l'interprétation du rôle et que la position paternelle a acquis une nouvelle légitimité (Boyer, Céroux, 2010 : 47), il apparaît malgré tout, d'une part que la volonté de certains hommes de s'affranchir de l'image traditionnelle du père est trop en avance par rapport aux représentations sociales de la famille et de la répartition genrée des tâches, encore dominantes, d'autre part que les femmes elles-mêmes, alors qu'elles ont intégré la sphère masculine du travail, laissent peu de possibilité d'entrée des hommes dans des espaces typiquement féminins, notamment ceux qui concernent la maternité.

\section{Bibliographie}

Akrich M., Pasveer B., Comment la naissance vient aux femmes, Paris, Mire-Synthelabo, 1996.

Benoît A., «Paroles d'hommes. Expérience d'un groupe de paroles pou hommes associé à la préparation à la naissance », in Marciano P. (coord.), Le père, l'homme et le masculin en périnatalité, Paris, Érès, 2003, p. 57-63.

Benoît A., « Naître père, quelle émotion ! « Tu seras un homme mon fils ! »... Même si je pleure ?», Spirale, 1/2005 ( $\left.n^{\circ} 33\right)$, p. 77-82.

Ben Soussan P., « Même les pères! », Spirale, 4/2003 ( $\left.{ }^{\circ} 28\right)$, p. 74-84.

14. Voir par exemple : Régnier-Loilier A., «L'arrivée d'un enfant modifie-t-elle la répartition des tâches domestiques au sein du couple ? », Population et sociétés, n 461, novembre 2009. 
Bessière Sabine, « La féminisation des professions de santé en France : données de cadrage », RFAS, 2005-1, p. 19-33.

Boyer D., Céroux B., « Les limites des politiques publiques de soutien à la paternité », Travail, genre et sociétés, novembre 2010 ( $\left.n^{\circ} 24\right)$, p. 47-62.

DRESS, « Les professions de santé au $1^{\text {er }}$ janvier 2011 », Séries Statistiques, nº 158 - juillet 2011. Delaisi De Parceval G., La part du père, Paris, Seuil, 1981.

Delaisi De Parceval G., Lallemand S., L'art d'accommoder les bébés, Paris, Odile Jacob, 1998 (1980).

Dolto F., Lorsque l'enfant paraît, Paris, Seuil, 1977.

Enquête nationale périnatale 2003. Compléments de cadrage : les disparités sociales en matière de santé périnatale et apports des autres sources, Ministère des Solidarités, de la Santé et de la Famille, février 2005.

Jacques B., Sociologie de l'accouchement, Paris, PUF-Le Monde, 2007.

INSEE, Médecins suivant le statut et la spécialité en 2012, Tableaux de l'économie française, 2012.

Hurstel F., La déchirure paternelle, Paris, PUF, 1996.

Laplantine F., Anthropologie de la maladie, Paris, Payot, 1992 (1986).

Leulliez M., "Ferdinand Lamaze et l'accouchement sans douleur ", Actes du colloque $50^{e}$ anniversaire de l'accouchement sans douleur, Châteauroux, septembre 2002.

Neyrand G., L'enfant, la mère et la question du père. Un bilan critique de l'évolution des savoirs sur la petite enfance, Paris, PUF, 2000.

This B., Le père, acte de naissance, Paris, Seuil, 1980.

Truc G., « La paternité en maternité. Une étude par observation », Ethnologie française, XXXVI, 2006, 2, p. 341-349.

Valabrègue C., La condition masculine, Paris, Payot, 1968. 